Psychotherapeut 2012 $\cdot 57: 201-203$

DOI 10.1007/s00278-012-0903-2

Online publiziert: 25. April 2012

(c) Springer-Verlag 2012

Anna Buchheim ${ }^{1}$. Manfred Cierpka ${ }^{2}$

${ }^{1}$ Institut für Psychologie, Universität Innsbruck, Österreich

${ }^{2}$ Institut für Psychosomatische Kooperationsforschung und Familientherapie, Universität Heidelberg

\title{
Neuronale Korrelate psychotherapeutischer Interventionen
}

ne-Persönlichkeitsstörung, posttraumatische Belastungsstörung). Die Autoren berichten von gängigen experimentellen Paradigmen in der funktionellen Bildgebung und spezifischen Forschungsfeldern (z. B. kognitive Defizite als Grundlage neuropsychologischer Therapien, Suche nach Neuroendophänotypen, Prädiktion von Therapieeffekten). Karch et al. fassen zusammen, dass bei Erkrankungen, bei denen Emotionen eine zentrale Rolle spielen (u. a. Depressionen, Angsterkrankungen, Borderline-Persönlichkeitsstörung), häufig Dysfunktionen in Hirnregionen auftreten, die mit der Regulierung von Emotionen assoziiert werden. Psychotherapeutische Interventionen könnten zu einer Art „Normalisierung“ der neuronalen Aktivität in diesen Bereichen führen (z. B. Amygdala, ventromedialer präfrontaler Kortex, anteriorer zingulärer Kortex, orbitofrontaler Kortex). Karch et al. schlussfolgern kritisch, dass bisher das Wissen über neurobiologische Grundlagen von psychotherapeutischen Interventionen noch unzureichend ist. Eine noch relativ geringe Zahl an Studien sowie methodische Probleme (u. a. kleine Stichproben, unzureichende Kontrollbedingungen, große Variabilität der verwendeten Verfahren) erschweren es, zuverlässige, generalisierende Aussagen zu treffen. Den Autoren erscheint in Zukunft die Untersuchung von unterschiedlichen Wirkfaktoren (auch Prozessfaktoren) bzw. Techniken innerhalb der Psychotherapie bedeutsam, da weitere Erkenntnisse zur Thera- pieprädiktion den therapeutischen Prozess maßgeblich beeinflussen könnten.

Die Originalarbeit von Köchel u. Schienle berichtet über die neuronalen Korrelate der Verhaltenshemmung auf emotionale Signale im Rahmen des Aufmerksamkeitsdefizit- und Hyperaktivitätssyndroms (ADHS). Neurowissenschaftliche Studien weisen auf ein Defizit im Erkennen von Emotionen des Gegenübers hin, das bei Kindern und auch bei Erwachsenen mit ADHS insbesondere bei Ärger stark ausgeprägt ist. Köchel u. Schienle greifen auf, wie neurowissenschaftliche Methoden dazu beitragen könnten, effizientere therapeutische Interventionen zu entwickeln. Da die Verbesserung affektiver Basisfertigkeiten intendiert ist, werden Verfahren benötigt, die Übungsmöglichkeiten bereitstellen. Nach Meinung der Autoren kann die emotionale Go-NoGo-Aufgabe hierfür ein wertvolles Tool darstellen. Eine Präsentation über den Computer ermöglicht, neues Wissen $\mathrm{zu}$ erwerben und $\mathrm{zu}$ trainieren, wobei der Patient vom Computer kontinuierlich Rückmeldung über seine Fortschritte erhält. Für die Arbeit mit Kindern steht der Spielaspekt im Zentrum. Im Erwachsenenbereich ist v. a. wichtig, das Verfahren gut in den Alltag der Patienten zu integrieren, sodass es nicht als zusätzliche Belastung erlebt wird. In der hier berichteten Pilotstudie zeigte sich, dass ADHS-Patienten von der wiederholten Übung der emotionalen Go-NoGo-Aufgabe profitierten und deren Übungsgewinne im ofDepressionen, Zwangsstörung, Borderli- 
fenen Verhalten direkt ablesbar sind. Eine begleitende Auswertung neuronaler Korrelate kann zudem Auskunft darüber geben, inwieweit sich die für die Aufgabe relevante Hirnaktivierung verändert. Die Autoren schließen mit dem Fazit, neurowissenschaftliche Ansätze im Bereich der ADHS-Störung stärker für die Entwicklung therapeutischer Komponenten und zur Abbildung von Therapieerfolgen heranzuziehen, um individuelle Bedürfnisse des Patienten im diagnostischen Prozess besser zu erfassen und individualisierte therapeutische Maßnahmen anbieten zu können.

Die Originalarbeit von Buchheim et al. beschreibt einen individualisierten Forschungsansatz im Bereich der Bildgebung, um erstmals neuronale Veränderungen bei chronisch depressiven Patienten während einer psychoanalytischen Psychotherapie mithilfe eines Bindungsparadigmas zu erfassen. Die neuronalen Befunde wurden kürzlich veröffentlicht (Buchheim et al. 2012, PLoS One 7:e33745) und werden in diesem Beitrag mit den Befunden der signifikanten Veränderung von Bindungsrepräsentationen nach 15-monatiger psychoanalytischer Behandlung zusammengefasst. In der Studie wurden chronisch-depressive Patienten ( $\mathrm{n}=16)$ am Anfang und nach 15 Monaten psychoanalytischer Therapie im Vergleich zu gesunden Kontrollprobanden ( $\mathrm{n}=17) \mathrm{mit}$ dem Adult Attachment Projective Picture System (AAP) interviewt, um die Bindungsrepräsentationen zu erfassen. Weiterhin wurden die Patienten und Probanden zu 2 Messzeitpunkten mithilfe der funktionellen Magnetresonanztomographie (fMRT) untersucht; hierbei wurden ihnen personalisierte Kernsätze aus den eigenen AAP-Interviews zusammen mit den AAP-Bildern präsentiert. Die persönlichen Sätze wurden mit neutralen, die Umgebung beschreibenden Sätzen kontrastiert. Die Patienten zeigten zu Beginn der Behandlung einen höheren Anteil an desorganisierten Bindungsrepräsentationen im Vergleich zu den Gesunden. Der unverarbeitete Bindungsstatuts der Patienten veränderte sich nach 15 Monaten zu organisierten Bindungsrepräsentationen. Neuronal war bei den Patienten zu Beginn der Behandlung eine höhere Aktivierung im linken anterioren Amygda-
la-Hippocampus-Komplex, im subgenualen Cingulum und im medialen präfrontalen Kortex als bei den Kontrollprobanden festzustellen. Diese erhöhte Aktivierung war nach 15 Monaten signifikant reduziert und glich sich den Gesunden an. Die verminderte neuronale Aktivierung korrelierte signifikant mit der Symptomverbesserung. Wie hier herausgearbeitet und deutlich gemacht wurde, plädieren die Autoren für einen Forschungsansatz mithilfe personalisierter Stimuli im Bereich der neurobiologischen Psychotherapieforschung, um unbewusste Aspekte zu erfassen, die für eine psychoanalytische Therapie von Relevanz sein könnten.

In einer Originalarbeit im Kontext der psychodynamischen Psychotherapie berichten Beutel et al. über erste Langzeitergebnisse einer fMRT-Studie an Panikpatienten, die in einer stationären Kurzzeittherapie behandelt wurden. In einem standardisierten Go-Nogo-Paradigma untersuchten die Autoren vor und nach Therapie Verhaltensmaße und zerebrale Aktivierung von 9 Panikpatienten auf affektive Wortreize mit bedrohlichen, neutralen und positiven Inhalten; in vergleichbarem Abstand wurden 18 gesunde Kontrollpersonen untersucht. In einer Langzeitkatamnese ca. 3 Jahre nach Abschluss der Therapie analysierten die Autoren den Einfluss der Hirnaktivierung vor und nach Therapie auf den Langzeitverlauf von panikbezogenen Ängsten und Körpersensationen. Erwartungsgemäß zeigte sich zu Behandlungsbeginn eine vermehrte limbische Aktivierung (Amygdala, Hippocampus), begleitet von einer verminderten präfrontalen Aktivierung, auf negative Wörter. Bei Therapieende verbesserte sich die Paniksymptomatik deutlich, und es normalisierte sich die frontolimbische Dysfunktion. Die verminderte Aktivierung des limbischen Systems unter der Therapie sagte auch günstigere Langzeitergebnisse vorher. Die Langzeitkatamnese ist als eine interessante Pilotstudie zu betrachten, an der 7 Patienten teilnahmen, von denen sich 4 noch in ambulanter psychotherapeutischer Behandlung (2 mit zusätzlicher Einnahme von Psychopharmaka) befanden.

Der Originalbeitrag von Schiepek et al. $\mathrm{zu}$ „Neurobiologische Korrelate der Psychotherapie von Zwangsstörungen“" be- schreibt neben dem Störungsbild und seinen Unterformen die wesentlichen neuropsychologischen Funktionsbeeinträchtigungen und die neuronalen Netzwerke, in denen zwangsspezifische Auffälligkeiten gefunden wurden. Hierbei handelt es sich um eine Erweiterung frontostriatothalamofrontaler Schaltkreise. Die Autoren fassen zunächst die wichtigsten Studien der neurobiologischen Psychotherapieforschung zusammen und zeigen Perspektiven für eine integrierte Prozessforschung auf. Eine eigene Prozess-Outcome-Studie fokussierte nicht nur auf Vorher-nachher-Effekte, sondern auf spezifische Merkmale des Veränderungsprozesses. Einbezogen wurden 9 nichtmedizierte Waschzwangpatienten und 9 Kontrollpersonen. Die stationäre Behandlung (Verhaltenstherapie, $z$. T. kombiniert mit anderen Therapieansätzen) dauerte im Mittel ca. 2 Monate. Die tägliche Dateneingabe (Selbsteinschätzungen) mit einem internetbasierten Monitoring-System (Synergetic Navigation System, SNS) resultierte in Zeitreihen mit durchschnittlich 55 Messzeitpunkten. Die visuelle Symptomprovokation wurde mit individuellen zwangsauslösenden Bildern sowie mit ekelauslösenden und neutralen Bildern aus dem International Affective Picture System (IAPS) für Patienten und gesunde Kontrollpersonen in gleicher Weise mit identischen Abständen zwischen den fMRT-Scans durchgeführt. Kasuistische Auswertungen und auch die Ergebnisse der Gesamtstichprobe weisen darauf hin, dass sowohl Therapieeffekte (Vorher-nachher-Vergleich) wie auch psychische Ordnungsübergänge mit spezifischen neuronalen Musterveränderungen korrelieren. Diese ließen sich u. a. im anterioren und medialen zingulären Kortex, im rechten lateralen frontalen Kortex, im Nucleus caudatus, in bestimmten Arealen des Motorkortex (z. B. im supplementärmotorischen Areal) sowie parietal-okzipital im Cuneus und Präcuneus lokalisieren. Nach Meinung der Autoren ließen sich in Zukunft das Neurofeedback mit EchtzeitfMRT und die nichtinvasive Neuromodulation zusammen mit Psychotherapie evtl. $\mathrm{zu}$ vielversprechenden Kombinationsansätzen ausbauen.

In diesen aktuellen Beiträgen zur Neurobiologie der Psychotherapie aus 
dem deutschsprachigen Raum erscheinen die folgenden Aspekte wichtig für die weitere Forschung: Die vorherrschende reine Outcome-Forschung sollte um den Aspekt der Prozessforschung im Therapieverlauf ergänzt werden. Wünschenswert wären zudem Follow-up-Messungen nach Therapieende und Langzeitbefunde. Die Stimulation sollte neben Standardmaterial auch individuelles und für den einzelnen Therapieprozess spezifisches Material enthalten, um einen individualisierten, personalisierten neurowissenschaftlichen Ansatz in der neurobiologischen Psychotherapieforschung zu gewährleisten

\section{Korrespondenzadresse}

Prof. Dr. Anna Buchheim Institut für Psychologie, Universität Innsbruck Innrain 52, 6020 Innsbruck Österreich

anna.buchheim@uibk.ac.at

\section{Schwerpunktthemen}

Das Herausgebergremium der Zeitschrift Psychotherapeut lädt Autorinnen und Autoren ein, an den geplanten Schwerpunkten mitzuarbeiten und geeignete Manuskripte einzureichen. Diese werden dem üblichen Reviewverfahren unterzogen. Darüber hinaus freuen wir uns über die Zusendung freier (unaufgeforderter) Originalia zu selbstgewählten Themen.

Bitte schicken Sie Ihren Beitrag an die Redaktion:

Regine.Karcher-Reiners@springer.com

Die Schwerpunktplanung ist vorläufig und kann kurzfristig umdisponiert werden.

Heft 6/12: Psychosenpsychotherapie

(Deadline 30.6.2012)

Heft 1/13: OPD

(Deadline 02.09.2012)

Heft 2/13: Burnout

(Deadline 02.11.2012)

Heft 3/13: Wie viel Verantwortung hat die Psychotherapie?

(Deadline 02.01.2013) 